\title{
Bourgain and Tao Awarded 2012 Crafoord Prize
}

JEAN BOURGAIN of the Institute for Advanced Study, Princeton and TERENCE TAO of the University of California, Los Angeles have been awarded the 2012 Crafoord Prize in Mathematics of the Royal Swedish Academy of Sciences "for their brilliant and groundbreaking work in harmonic analysis, partial differential equations, ergodic

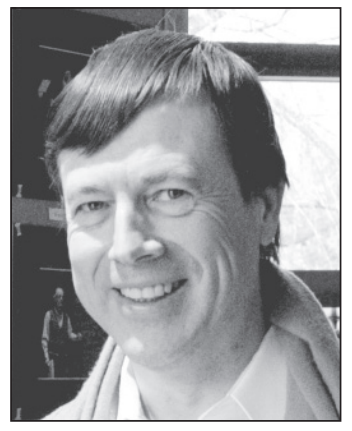

Jean Bourgain

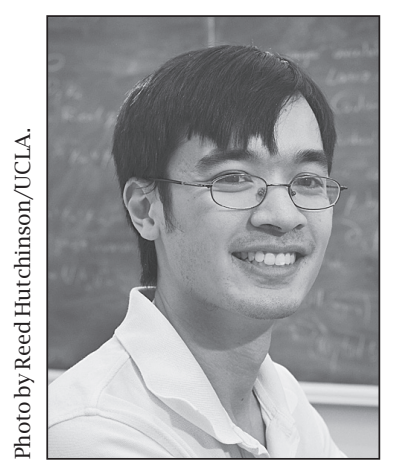

Terence Tao theory, number theory, combinatorics, functional analysis and theoretical computer science."

The prize citation reads in part: “This year's Crafoord Prize Laureates have solved an impressive number of important problems in mathematics. Their deep mathematical erudition and exceptional problem-solving ability have enabled them to discover many new and fruitful connections and to make fundamental contributions to current research in several branches of mathematics. On their own and jointly with others, Jean Bourgain and Terence Tao have made important contributions to many fields of mathematicsfrom number theory to the theory of nonlinear waves. The majority of their most fundamental results are in the field of mathematical analysis. They have developed and used the toolbox of analysis in groundbreaking and surprising ways. Their ability to change perspective and view problems from new angles has led to many remarkable insights, attracting a great deal of attention among researchers worldwide."

Bourgain has proved several groundbreaking results on well-posedness of nonlinear differential equations, such as the Schrödinger equation of quantum mechanics and the Korteweg-de Vries equation of wave propagation. These theorems

Elaine Kehoe is contributing writer for the Notices. Her email address is e 1aine 1 kehoe@cox.net.

DOI: http://dx.doi.org/10.1090/noti840 guarantee that the solutions to these equations evolve in a unique way, no matter how irregular the initial state of the system might be. He has made contributions in many areas, such as his 2010 result in Apollonian gaskets-infinite collections of circles that just touch without overlapping. In these arrangements, the radius of each bubble is 1 divided by an integer. Together with Elena Fuchs, Bourgain showed that, if you pick any integer $n$, there is a nonzero probability that some bubble in the Apollonian foam has radius equal to $1 / n$. Bourgain was awarded the Fields Medal in 1994 and has also been awarded the Salem Prize (1983), the Damry-Deleeuw-Bourlart Prize (Belgian Science Prize, 1985), the Langevin (1985) and Cartan (1990) Prizes of the French Academy, the Ostrowski Prize (1991), and the Shaw Prize (2010).

Tao is a world-renowned mathematician working in a number of branches of mathematics, including harmonic analysis, partial differential equations, combinatorics, number theory, and signal processing. He is known for his highly original solutions of very difficult and important problems and for his technical brilliance in the use of the necessary mathematical machinery. He and Ben Green proved that there are arbitrarily long arithmetic progressions of prime numbers - a result now known as the Green-Tao theorem-which had been a major unsolved problem in number theory for centuries. He is also known for such practical work as his invention, with Emmanuel Candes, of compressed sensing. His areas of research include harmonic analysis, partial differential equations, combinatorics, and number theory. He received the Fields Medal in 2006 for his contributions in these fields. He has held Sloan Foundation (1999-2001), Packard (1999-2006), and MacArthur (2007) fellowships. He has also received the Salem Prize (2000), the Bôcher Prize (2002), the SASTRA Ramanujan Prize (2006), the Ostrowski Prize (2007), the Waterman Award (2008), and the King Faisal Prize (2010).

"Bourgain and Tao have similar and very unique strengths," says Peter Sarnak of the Institute for 
Advanced Study. "Their ability to see through technical difficulties that prevent almost anyone else to move forward has allowed them to resolve long-standing problems in an amazingly diverse set of areas."

They will share the monetary award of four million Swedish kronor, approximately US\$580,000.

The Anna-Greta and Holger Crafoord Fund was established in 1980, and the first prize was awarded in 1982. The prize is intended to promote international basic research in the disciplines of astronomy, mathematics, the geosciences, the biosciences (with particular emphasis on ecology), and polyarthritis (rheumatoid arthritis).

Previous recipients of the Crafoord Prize in Mathematics are Maxim Kontsevich and Edward Witten (2008), Alain Connes (2001), Simon Donaldson and Shing-Tung Yau (1994), Pierre Deligne and Alexander Grothendieck (1988), and V. I. Arnold and Louis Nirenberg (1982).

- Elaine Kehoe

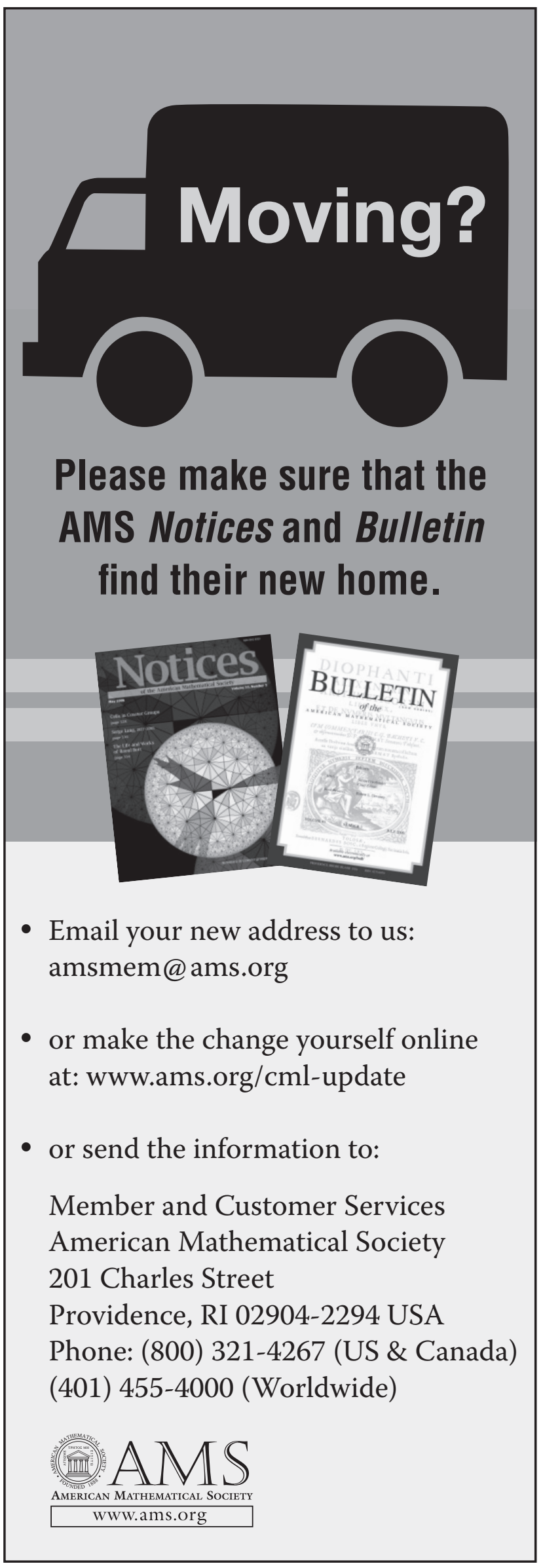

